\title{
Reflets
}

Revue ontaroise d'intervention sociale et communautaire

\section{Troisième âge en action}

\section{Hélène Carrière et Suzanne Poirier}

Volume 2, numéro 2, automne 1996

Vieillir à l'aube de l'an 2000

URI : https://id.erudit.org/iderudit/026136ar

DOI : https://doi.org/10.7202/026136ar

Aller au sommaire du numéro

Éditeur(s)

Reflets : Revue ontaroise d'intervention sociale et communautaire

ISSN

1203-4576 (imprimé)

1712-8498 (numérique)

Découvrir la revue

Citer cet article

Carrière, H. \& Poirier, S. (1996). Troisième âge en action. Reflets, 2(2), 138-143.

https://doi.org/10.7202/026136ar

Tous droits réservés (C) Reflets : Revue ontaroise d'intervention sociale et communautaire, 1996

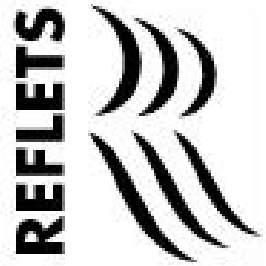

Ce document est protégé par la loi sur le droit d'auteur. L'utilisation des services d’Érudit (y compris la reproduction) est assujettie à sa politique d'utilisation que vous pouvez consulter en ligne.

https://apropos.erudit.org/fr/usagers/politique-dutilisation/ 


\section{Troisième âge en action}

Hélène Carrière, infirmière et Suzanne Poirier, infirmière auxiliaire,

Liaison Santé Vanier, Programme des aînés, Service de la santé d'OttawaCarleton, Ottawa

À maintes reprises, des personnes aînées de la communauté de Vanier ont révélé "qu'être en action» physiquement et mentalement est le secret de leur énergie et de leur santé! Liaison Santé Vanier est un programme du Service de la santé d'OttawaCarleton, qui prône la promotion de la santé par et pour les aînés francophones. La mobilisation communautaire constitue un élément clef des activités de ce programme. Dans cet article, les auteures traiteront de l'historique de ce programme, de son évolution, de ses principales activités, des caractéristiques des personnes aînées qui s'y impliquent, du rôle des intervenantes et de son orientation future.

\section{Historique}

En 1988, le Service de la santé d'Ottawa-Carleton entreprit une consultation de grande envergure et une planification, avec la participation de membres du personnel du Service de la santé, $\mathrm{du}$ Conseil sur le vieillissement et d'agences communautaires en vue d'identifier les lacunes dans les services aux personnes ainées de la communauté, et de déterminer le rôle du Service de la santé face à ces lacunes.

Le résultat de ce processus fut publié dans un document intitulé: Réalisons notre potentiel: Plan directeur des programmes pour per- 
sonnes âgées du Service de la santé. Dans ce document, neuf stratégies majeures ont été identifiées, et le développement d'un programme nommé Liaison Santé a été classé comme première stratégie.

Liaison Santé a pris son envol en juillet 1989, dans une communauté anglophone rurale d'Ottawa-Carleton et dans Vanier, communauté à prédominance francophone. Deux ans plus tard, le programme s'étendit à cinq autres communautés rurales et à une autre communauté francophone.

\section{Évolution}

Le principe de base du programme Liaison Santé devait être un travail de collaboration entre l'intervenante du Service de la santé et un comité aviseur formé de personnes aînées de la communauté. Toutefois, la démarche suivie pour composer le comité consultatif n'a pas nécessairement été la même d'une communauté à l'autre.

À Vanier, l'infirmière coordonnatrice de Liaison Santé à rencontré le directeur du Centre des services communautaires afin d'initier le contact avec la communauté, et d'établir progressivement la crédibilité du programme. Le directeur exprima sa vision de Vanier et de ses citoyens du troisième âge. Il discuta du rôle du Centre et du partenariat possible avec Liaison Santé.

Par la suite, la location d'un local fut offerte par le Centre des services communautaires de Vanier. Cette initiative s'est avérée profitable, puisqu'elle a permis aux membres de la communauté de Vanier d'avoir sur place une intervenante santé.

La prochaine étape fut pour l'infirmière de rencontrer les personnes aînées dans leurs clubs sociaux. Au début, le contact se faisait de façon informelle, tout simplement pour les connaitre et être à l'écoute de leurs besoins. Peu à peu, les personnes aînées s'aventurèrent à discuter de solutions possibles face aux besoins exprimés. 
Enfin, comme chaque club social était affilié à une église, l'infirmière consulta un groupe par paroisse afin de déterminer les priorités d'action. Les thèmes proposés furent: l'isolement, le soin des pieds et le deuil. Il fallait maintenant agir. Un comité consultatif de huit personnes, représentant des clubs sociaux ou la communauté en général, a été constitué. Les membres du comité se dirent intéressés à participer à tous les niveaux de la planification des activités de Liaison Santé. De plus, ils joueraient un rôle de premier plan dans la diffusion de l'information auprès des autres membres de leurs clubs et de la communauté. Le noyau de base de Liaison SantéVanier était maintenant une réalité!

Le comité consultatif Liaison Santé Vanier 1996 est maintenant composé de sept personnes aînées, dont certaines sont des doyennes du programme. Nous avons constaté que les membres expriment davantage leurs besoins et apportent plus de suggestions qu'auparavant. La confiance en soi a augmenté, grâce à de petites et grandes réussites, à la visibilité plus accentuée, à de nouvelles expériences, à la prise en charge accrue et à la fierté du groupe.

\section{Activités}

Le choix des activités de Liaison SantéVanier complète le mandat du Service de la santé face aux besoins des personnes aînées. Heureusement, l'expérience a prouvé que les besoins de la communauté correspondent souvent aux priorités du Service de la santé. Ainsi, l'exercice, le soin des pieds, la nutrition, l'isolement, la sécurité, le deuil, le transport, la santé émotionnelle figurent parmi les préoccupations majeures établies au cours des années par le comité consultatif Liaison Santé.

Conférences, dîners-causeries, groupes d'exercice, séances "cuisiner pour soi», ateliers de découverte de soi ont tour à tour été des moyens de promouvoir la santé. Cependant, le Comité consultatif Liaison Santé Vanier a aussi innové dans ses choix de 
stratégies: participation à des entrevues et vidéos à la télévision communautaire, travail concernant la sécurité, théâtre Défi-santé, Foire nutrition impliquant neuf restaurateurs de la communauté, forums pour personnes aînées, implication dans la rédaction d'un livre souvenir pour les gens du troisième âge de Vanier!

Pour mettre en œuvre ces stratégies innovatrices, les membres du comité consultatif ont collaboré à la rédaction de textes pour le montage de vidéos, ont joué le rôle de la personne interviewée, sont devenus comédiens dans une pièce de théâtre, se sont déplacés dans la rue pour inspecter la sécurité, ont apporté leurs suggestions dans la planification d'activités impliquant plusieurs partenaires de la communauté de Vanier, et ont immortalisé des souvenirs dans un livre intitulé : «En passant ... par l'Ontario» travail fait en collaboration avec le programme d'alphabétisation: La magie des lettres de Vanier.

Nous avons constaté qu'à travers ce bourdonnement d'activités, les membres du comité consultatif ont, avec les années, accru leur confiance en soi, et sont sortis des sentiers battus pour promouvoir la santé auprès du plus grand nombre d'aînés possible.

\section{Caractéristiques des participants}

Nous pouvons dire que les caractéristiques des ainés membres du comité consultatif Liaison SantéVanier sont les suivantes: des bénévoles de longue date, des personnes attachées à leur communauté et à leur culture, impliquées dans un club d'ainés ou une association, ayant le goût d'apprendre et d'agir, le sens des responsabilités et désirant se tenir à jour dans le but de maintenir et d'améliorer leur santé.

Ces personnes ont l'esprit ouvert et sont généreuses de leur temps. De plus, elles transmettent les messages à leurs clubs et associations et protègent leurs droits. 
Enfin, les membres du comité consultatif sont fiers d'appartenir à ce groupe. Leur plus grande richesse est fort probablement leur sagesse, acquise au long de leur expérience de vie.

\section{Rôle des intervenantes}

Le document Liaison Santé, tous ensemble pour la santé des aînés précise bien les attentes et les rôles à l'égard des intervenantes et des professionnelles de la santé:

«En effet, à tour de rôle, il/elle devra être la personne aidante, la personne ressource, un médiateur, un facilitateur et un partisan des intérêts communautaires. Le professionnel de la santé se doit de reconnaître les ressources du groupe, d'accepter leur expérience comme étant valable et possédant une valeur égale à son expertise». (Levesque et Hughes 1989:9)

Les intervenantes peuvent aussi servir de lien avec d'autres partenaires dans la communauté, afin d'augmenter la visibilité de Liaison Santé Vanier et amener le groupe vers de nouvelles expériences et initiatives. Finalement, les intervenantes doivent pousser les gens à l'action par leur enthousiasme et leur conviction. Elles partagent avec le groupe les joies des succès et soutiennent aussi le groupe en cas d'échec.

\section{Orientation future}

Les étapes suivantes chercheront à favoriser une plus grande prise en charge par le groupe, par le biais des responsabilités à endosser, par la confiance bâtie pour faire valoir son point de vue, par l'expertise acquise en matière de leadership, par l'ouverture face 
aux alliances avec d'autres partenaires dans la communauté et par le contexte socio-économique.

\section{Conclusion}

Dans cet article, nous avons traité de la mise sur pied de Liaison Santé Vanier, de son évolution, de ses principales activités, des caractéristiques des aînés qui s'y impliquent, du rôle des intervenantes et de son orientation future. À l'aube de l'an 2000, cette initiative vise à donner aux aînés une autonomie accrue, à veiller au maintien de leur santé physique et intellectuelle, et à leur donner la fierté de participer à l'action pour prendre la place qui leur revient.

\section{Bibliographie}

LA MAGIE DES LETTRES, (1996), «En passant ... par l'Ontario».

LEVESQUE, Johanne et Lorette HUGHES, (1989), «Liaison Santé: Tous ensemble pour la santé de nos aînés", 1-10.

SERVICE DE LA SANTÉ D’OTTAWA-CARLETON, (1988), Réalisons notre potentiel: Plan directeur des programmes pour personnes âgées du Service de la santé, Ottawa, Service de la santé d'OttawaCarleton.

SERVICE DE LA SANTÉ D'OTTAWA-CARLETON, (1990), Health Connection: A Community Development Strategy, Ottawa, Service de la santé d'Ottawa-Carleton.

SERVICE DE LA SANTÉ D'OTTAWA-CARLETON, (1993), Health Forum Presentation, Health Connection/Liaison Santé, Ottawa, Service de la santé d'Ottawa-Carleton.

SERVICE DE LA SANTÉ D'OTTAWA-CARLETON, (1995), Si je savais comment ... Rejoindre les francophones, faut l'faire, Ottawa, Service de la santé d'Ottawa-Carleton. 